\title{
Dust clouds or magnetic spots? Exploring the atmospheres of $L$ dwarfs with time-resolved spectrophotometry
}

\author{
C. A. L. Bailer-Jones \\ Max-Planck-Institut für Astronomie, Königstuhl 17, 69117 Heidelberg, Germany
}

Received 27 March 2002 / Accepted 15 May 2002

\begin{abstract}
I present the results of a program to spectrophotometrically monitor the L1.5 dwarf 2MASSW J1145572+231730 to identify the cause of photospheric variability in ultra cool dwarfs. Plausible candidates are magnetically-induced star spots and inhomogeneous photospheric dust clouds. Based on the atmospheric models and synthetic spectra of Allard et al. (2001), the expected signatures of these phenomena in the 0.5-2.5 $\mu \mathrm{m}$ wavelength region are presented and discussed. Near infrared spectra of 2M1145 were obtained along with a nearby reference star observed simultaneously in the spectrograph slit. No convincing variability over a 54 hour interval is found in any one of several colour indices designed to be most sensitive to dust- and spot-related variability. Nonetheless, a significant correlation between the variability of two colour indices is found. This is slightly more consistent with the dust-related variability model than the cool spot one considered. Based on the theoretically predicted signatures and the median errors in the colour indices (0.03-0.05 mag), upper limits are placed on the coverage of possible spots and clouds. Assuming the L dwarf to be best modelled by a dusty atmosphere at $1900 \mathrm{~K}$, coherent clear clouds are limited to a coverage of $10-15 \%$ of the projected surface area and $200 \mathrm{~K}$ cooler spots to a $20 \%$ coverage. A larger coverage of many small features varying incoherently cannot be ruled out with this method. A lower effective temperature restricts coherent clear clouds to be much smaller; a higher temperature allows both clouds and spots to be larger. These upper limits are consistent with the two separate variability detections in the $I$-band reported by Bailer-Jones \& Mundt (2001).
\end{abstract}

Key words. stars: atmospheres - stars: low-mass, brown dwarfs - stars: starspots - stars: variables: general stars: individual: 2MASSW J1145572+231730 - methods: data analysis

\section{Introduction: variability, dust and magnetic fields in ultra cool dwarfs}

In a study of 21 low mass stars and brown dwarfs, BailerJones \& Mundt (2001; hereafter BJM) found evidence for $I$-band photometric variability in $11 \mathrm{M}$ and $\mathrm{L}$ dwarfs, with variability timescales of a few hours and rms amplitudes of 0.01-0.06 mags. In a few cases, this variability could not be ascribed to a rotational modulation by stable surface features (e.g. cool spots), such as those often found on premain-sequence, earlier-type stars (e.g. Herbst et al. 2001). This observed non-periodic variability was therefore interpreted as evidence for the evolution of surface features on a timescale shorter than a rotation period. This could happen if the features rapidly form and dissipate or if they migrate with respect to the rotation.

This raised the question as to the physical nature of the putative surface features on ultra cool dwarfs (UCDs) ${ }^{1}$.

\footnotetext{
* e-mail: calj@mpia-hd.mpg.de
}

BJM found no correlation between the presence or amplitude of variability and the $\mathrm{H} \alpha$ equivalent width (although many of the objects had no $\mathrm{H} \alpha$ data). Taking the latter as a measure of chromospheric activity and hence as a proxy for the size and/or temperature contrast of magneticallyinduced photospheric spots, this lack of correlation provides evidence against the surface features being magnetic spots. This is supported by the observation of Gizis et al. (2000), who found that the ratio of $\mathrm{H} \alpha$ emission to bolometric luminosity decreased by more than two orders of magnitude from M7 to L1. As BJM also found that variability appeared to be more common later than M9, they

\footnotetext{
${ }^{1}$ An ultra cool dwarf (UCD) is loosely defined here as any dwarf star with spectral type later than about M 8, thus including $\mathrm{L}$ and $\mathrm{T}$ dwarfs. UCDs have effective temperatures below about $2800 \mathrm{~K}$, surface gravities in the range $300-3000 \mathrm{~m} \mathrm{~s}^{-2}$ (i.e. $\log g$ of $4.5-5.5$ ) and masses less than about $0.1 M_{\odot}$. Following the mass definition of substellar objects, a given UCD could be a low mass hydrogen-burning star, a brown dwarf or a gas giant planet.
} 
concluded that their observed variability was probably not magnetic in nature.

It has now been well established that dust grains form in the atmospheres of UCDs, and that at least some component of these remain suspended in the photosphere at effective temperatures above about $1800 \mathrm{~K}$ (Burrows \& Sharp 1999; Lodders 1999; Chabrier et al. 2000; Allard et al. 2001; Schweitzer et al. 2001; Marley et al. 2002). The presence of solid condensates combined with the fact that UCDs are probably fully convective (Chabrier \& Baraffe 2000) and are often rapid rotators (Basri et al. 2000 ), provides a mechanism for turbulent processes to drive dust dynamics and produce time-dependent opacities. BJM took this to be the single most likely mechanism to explain their observed variability.

However, doubt about the general absence of magnetic activity in $\mathrm{L}$ dwarfs has been raised by the detection of radio emission in four UCDs (of a sample of 13) by Berger (2002) and Berger et al. (2001). From the intensity of the continuous emission and flaring at $8.5 \mathrm{GHz}$ detected in these objects, Berger (2002) concludes that the emission mechanism is most likely synchrotron and not thermal, indicating the presence of magnetic fields and electron column densities similar to those inferred for earlier-type flaring $\mathrm{M}$ dwarfs. Although based on a very small number of detections, these data also hint that the radio emission is no longer correlated with the $\mathrm{H} \alpha$ emission in the M7-L1 spectral type region where the latter decreases sharply, the implication being that $\mathrm{H} \alpha$ emission no longer traces magnetic activity.

While magnetic fields may well be present in UCDs, this does not necessarily mean that they produce photospheric spots. UCDs have very cool atmospheres and low ionization fractions (e.g. Meyer \& Meyer-Hofmeister 1999; Fleming et al. 2000). Thus there are relatively few free electrons and so little coupling between the gas and any magnetic field, with the result that cool spots may not be able to form in the visible photosphere. Gelino et al. (2002) have attempted to quantify this lack of coupling by showing that the magnetic Reynolds number is probably very $\operatorname{low}^{2}(<1)$ for UCDs with convective velocities below $100 \mathrm{~m} \mathrm{~s}^{-1}$. This neutrality of UCD photospheres can be reconciled with the relatively high electron column densities inferred by Berger (2002) on the assumption that the radio emission is primarily due to a high density of coronal electrons. In their own $I$-band monitoring of $18 \mathrm{~L}$ dwarfs, Gelino et al. (2002) found seven to be variable, and concluded on the basis of the neutrality of $\mathrm{L}$ dwarfs that the variability was not magnetic in origin. Further, Martín et al. (2001) detected photometric variability in the field M9 dwarf BRI0021-0214, which generally shows very weak magnetic activity, although an

\footnotetext{
${ }^{2}$ However, spots could still form deeper in the atmosphere where the Reynolds number is higher due to the lower ionization, and these may be visible if the atmosphere is less opaque due to the precipitation of dust (see Sect. 3.1).
}

$\mathrm{H} \alpha$ flare has been observed from this object (Reid et al. 1999).

This returns us to the behaviour of dust. In a cool static atmosphere, dust grains will form, grow and gravitationally settle below the photosphere creating a dustcleared photosphere depleted in those elements locked into the grains. By contrast, in a dynamic atmosphere, convection will provide a bouyancy acting against precipitation, and deep convection may recycle precipitated elements back into the visible atmosphere, thus retaining a dusty composition. First attempts to consider the relative efficiency of dust settling and convective recycling - and hence the vertical distribution of dust grains in the photosphere - have been made by Tsuji (2001) and Ackerman \& Marley (2001). A gradual transition from a dusty to a dust-cleared atmosphere is predicted to occur with decreasing effective temperature. Dust can be considered to be confined to a thin layer, the depth (pressure) of the base of which is determined by the condensation temperature of grains. Condensation can occur throughout the atmosphere above this base with increasing efficiency at higher altitudes (due to lower temperatures), although with decreasing contribution to the opacity on account of the decreasing density. A limit on the vertical extent of this layer may be set by the growth of grains above some critical size, at which point gravity overcomes turbulent support and these grains are precipitated out into deeper layers of the atmosphere. As the effective temperature decreases, the base of this dust layer moves to deeper parts of the atmosphere, where the integrated gas opacity (as seen from outside the photosphere) is increased. Hence the dust layer becomes less visible with the result that the spectrum increasingly represents a dust-cleared atmosphere. Such a mechanism may explain the switch from red to blue infrared colours for the transition from mid L dwarfs to $\mathrm{T}$ dwarfs.

Neither the model of Tsuji (2001) nor that of Ackerman \& Marley (2001) addresses the possibility of horizontal inhomogeneities or dust grain dynamics which would be required to produce variability in the unresolved disk (but see Helling et al. 2001). In a dusty or dust-cleared atmosphere, these inhomogeneities could be regions of enhanced dust formation and dust opacity, perhaps due to a localized deep convective column bringing dust forming material up from the deeper, opaque layers of the atmosphere. Alternatively, in a dusty atmosphere, dust formation may be slow in the material brought up by deep convection, thus creating a short-lived dust-free cloud.

\section{Current objectives and target information}

In the present paper, I report the results of an observational program to investigate further the physical cause of variability in UCDs. Such an investigation is naturally rather speculative given how little we known of timedependent atmospheric processes in UCDs. In light of this, I decided to spectrophotometrically monitor a UCD over a wide wavelength range. The near infrared (NIR) region 
$(0.9-2.5 \mu \mathrm{m})$ was chosen over the optical $(0.3-1.0 \mu \mathrm{m})$ because UCDs are significantly brighter there.

At the time of target selection (July 1999), the only known photometrically variable UCDs were the L1.5 dwarf 2MASSW J1145572+231730 (Bailer-Jones \& Mundt 1999) and the M9 dwarf LP944-20 (Tinney \& Tolley 1999). The former was selected because (1) we intended to monitor it again in the $I$-band and (2) it has a nearby bright reference star which can be used to obtain differential spectrophotometry. Due to rapid changes in sky transmission and brightness in the NIR, it was felt that spectrophotometry from regular calibration against standards was not viable. Instead, spectrophotometry is done relative to a second star in the same slit, on the assumption that this reference star is photometrically stable to some required level on the timescale of interest. This reference star (which is not presently in NED or SIMBAD and is saturated in the data of BJM) has designation 2MASSI $\mathrm{J} 1145560+231613$, and has $B, R, J, H$ and $K_{\mathrm{s}}$ magnitudes of $14.80,14.10,13.57,13.28$ and 13.18 respectively, as taken from the 2MASS second incremental release catalogue. These are most consistent with an unreddened or slightly reddened mid- or late-type $\mathrm{F}$ dwarf. The Galactic latitude of the field is $l=226^{\circ}, b=75^{\circ}$, implying that it is probably an older thick disk or halo star at $1-2 \mathrm{kpc}$, rather than a very young potentially variable star or pulsating supergiant.

2MASSW J1145572+231730 (hereafter 2M1145) is an L1.5 dwarf discovered by 2MASS with $I, J, H$ and $K_{\mathrm{s}}$ magnitudes of 18.6, 15.37, 14.52 and 13.92 respectively (Kirkpatrick et al. 1999; the $J, H, K_{\mathrm{s}}$ magnitudes are from the 2MASS second incremental release catalogue). The optical spectrum of Kirkpatrick et al. (1999) shows an $\mathrm{H} \alpha$ emission line with an equivalent width of $4.2 \AA^{3}$. The absence of strong lithium absorption implies that it may be a low mass star rather than a brown dwarf, but its late spectral type and apparent relative youth $(<1 \mathrm{Gyr})$ mean that it probably has a mass close to the stellar/substellar boundary at $0.075-0.08 M_{\odot}$.

Based on data taken in January 1999, Bailer-Jones \& Mundt (1999) found 2M1145 to be photometrically variable in the $I$-band with an RMS amplitude of 0.038 mags and a tentative period of 7.1 hours. An improved analysis of these data confirmed the detection of a significant variability - albeit with a slightly smaller amplitude (0.031 mags) - but did not confirm the period (BJM). New data taken simultaneously with that in the present paper (February 2000), and reported in BJM, made a much more significant detection of variability (amplitude 0.020 mags), again over a timescale of a few hours. The nature of the power spectra, plus the significant differences between the 1999 and 2000 power spectra, led BJM to conclude that the surface features evolved over a timescale of a few hours (see Sect. 1). Since then, variability has been discovered

\footnotetext{
3 The statement on p. 507 of Basri (2000) that 2M1145 has shown flaring in $\mathrm{H} \alpha$ is incorrect. The paper referenced cites flaring in a different object.
}

in a number of other late $\mathrm{M}$ and early to mid $\mathrm{L}$ dwarfs, some also showing non-periodic variability (Martín et al. 2001; Clarke et al. 2002; Gelino et al. 2002) and water variability has been claimed in a $\mathrm{T}$ dwarf (Nakajima et al. 2000).

The layout of the rest of this paper is as follows. In the next section I describe the observational signatures of different types of UCD atmospheric variability, based on the atmospheric models and synthetic spectra of Allard et al. (2001). In then describe the observational procedure used (Sect. 4) followed by the method of spectral extraction and combination (Sect. 5). The variability analysis is presented in Sect. 6 and the implications of these results for atmospheric changes in UCDs is given in Sect. 7. While no convincing variability signal was detected, the data nonetheless permit various extreme cases to be ruled out. The main points of the paper are summarised in Sect. 8 .

\section{Observability of theoretical predictions of atmospheric features}

\subsection{Method and models}

Predictions of the photometric signatures of surface features on UCDs can be obtained from combining synthetic spectra which represent (a) the "background" (featurefree) atmosphere and (b) the feature, in proportion to the surface coverage of the feature. For this purpose I will use the AMES-dusty and AMES-cond models of Allard et al. (2001). Both models permit dust grains to form, but they represent the extreme cases of dust precipitation. In the AMES-dusty model (hereafter dusty), precipitation is prevented (e.g. convective recycling is very efficient), whereas in the AMES-cond model (hereafter cond) the gravitational settling of dust efficiently clears the atmosphere of all condensed dust (and of course its constituents). In reality, intermediate situations probably exist with varying degrees of dust precipitation (e.g. Tsuji 2001; Ackerman \& Marley 2001). Therefore, the following predictions serve to examine the extreme effects which may occur and which can be observationally tested.

Both the dusty and cond models of Allard et al. (2001) assume that spherical dust grains form in chemical equilibrium with the gas phase. In the cond model this dust is removed from the atmosphere. The authors assume an interstellar size distribution of dust grains between 0.006 and $0.240 \mu \mathrm{m}$. They acknowledge that this is somewhat arbitrary, but provided that the largest particles are sufficiently small such that scattering remains in the Rayleigh regime for the wavelengths considered $(\lambda>0.5 \mu \mathrm{m})$, this is of no relevance to the formation of the spectral energy distribution. Convection is treated using the mixing length theory with a mixing length equal to the pressure scale height and a microturbulence velocity of $2 \mathrm{~km} \mathrm{~s}^{-1}$. While convection is included for calculating the energy transport, it is not included in the determination of the dust number densities. So in these models, convection is not a mechanism for returning precipitated dust elements back to the photosphere. 


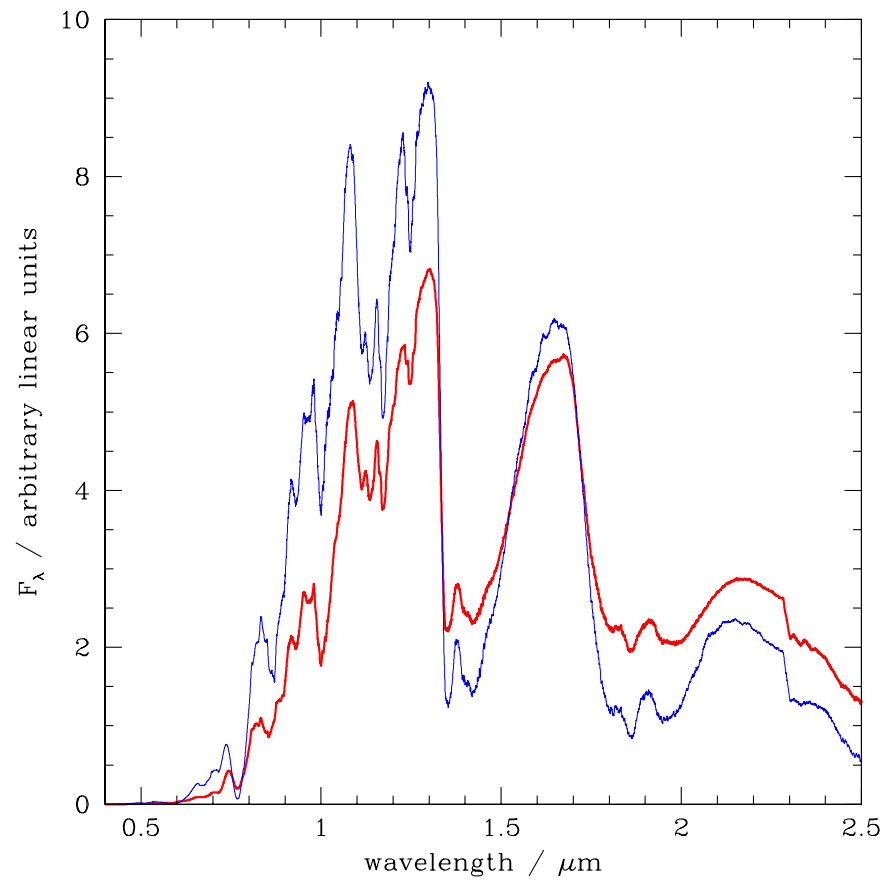

Fig. 1. AMES-dusty (thick/red line) and AMES-cond (thin/blue line) synthetic spectra from Allard et al. (2001) for $T_{\text {eff }}=1900 \mathrm{~K}$ and $\log g=5.5$, the nominal parameters for the L1.5 field dwarf 2M1145. These spectra, as well as those in Figs. 2-5, are shown at a resolution of $0.01 \mu \mathrm{m}$.

In the following, I consider a cloud on a dusty atmosphere to be a compact region cleared of dust (i.e. represented by the cond model) in thermal equilibrium with the "background" atmosphere, that is, at the same effective temperature. Clearly this assumption would not hold if material is brought up rapidly from deep within the star to form the cloud. Likewise, a cloud on a cond atmosphere is represented by the dusty model, again at the same effective temperature as the background atmosphere. Magnetic spots are modelled as compact regions with the same atmosphere (dusty or cond) as the background atmosphere, but with a lower effective temperature.

Of course, other causes of photometric variability in UCDs may exist. Newly formed UCDs may have accretion disks, infall of matter onto which could create observable hot spots, as could interactions with a close companion. However, magnetic fields, dust and atmospheric dynamics are ubiquitous, so a search for the observational signatures of these seems a sensible first step.

\subsection{Cloud and spot signatures at $1900 \mathrm{~K}$}

Based on the fits of the Allard et al. (2001) synthetic spectra to low and high resolution observations of $\mathrm{L}$ dwarfs by Schweitzer et al. (2001), an L1.5 spectrum (the spectral type of $2 \mathrm{M} 1145$ ) is most consistent with a dusty model with $T_{\text {eff }}=1900 \mathrm{~K}$ and $\log g=5.5$, although models with $\pm 100 \mathrm{~K}$ and \pm 0.5 dex in $\log g$ are equally valid depending on which data are used. Figure 1 shows this model spectrum from Allard et al. (2001), plus the cond spectrum at the same temperature and gravity for comparison. Dusty models explain well the NIR colours of UCDs down to mid L dwarfs (circa $1800 \mathrm{~K}$ ) (e.g. Chabrier et al. 2000). At $T_{\text {eff }}=1900 \mathrm{~K}$, dust grains form deep in the photosphere with the result that the photosphere is heated by several hundred Kelvin and the whole photosphere is moved "up" in the atmosphere, i.e. to lower gas pressures (cf. Figs. 5 and 6 of Allard et al. 2001). This dust opacity suppresses the flux blueward of about $1.3 \mu \mathrm{m}$ and leads to it being re-radiated at longer wavelengths. At these longer wavelengths, water steam is the dominant source of opacity, as can be seen from the deep water absorption bands at $1.35-1.45$ and $1.8-2.0 \mu \mathrm{m}$ in Fig. 1. The cond model atmospheres, by contrast, are more transparent and cooler, due to the lack of both dust grains and the molecular species which were locked into the dust.

Figure 2 shows the change in a $1900 \mathrm{~K}$ dusty spectrum due to the formation of a cond cloud covering $10 \%$ of the surface (thick/red solid line). It is shown in terms of the conventional magnitude system, such that the ordinate is

$\Delta m(\lambda)=-2.5 \log _{10}\left(\frac{e F_{\mathrm{c}}(\lambda)+(1-e) F_{\mathrm{a}}(\lambda)}{F_{\mathrm{a}}(\lambda)}\right)$

where $F_{\mathrm{a}}(\lambda)$ is the model spectrum $\left(\mathrm{W} \mathrm{m}^{-2} \AA^{-1}\right)$ of the cloud-free (background) atmosphere, $F_{\mathrm{c}}(\lambda)$ the spectrum of the cloud, and $e$ the cloud covering fraction, in this case 0.1. Figure 2 also shows this for a dusty cloud on a cond atmosphere (thin/blue solid line). Thus during a period of cloud formation and dissipation, the spectrum is predicted to change by the amount shown.

The formation of a cond cloud on a dusty atmosphere increases the flux over almost all of the region $0.5-1.3 \mu \mathrm{m}$ due to the reduced total dust opacity in the integrated disk in this wavelength region. Likewise, the lower temperature of the cond cloud results in the recombination of more water molecules and thus a larger opacity at wavelengths above $1.3 \mu \mathrm{m}$.

Another consequence is an increase in the strength of the absorption lines of the neutral alkali elements ( $\mathrm{Li}, \mathrm{Na}, \mathrm{K}, \mathrm{Rb}, \mathrm{Cs}$ ). This occurs because the lower temperature cond cloud decreases the opacity below these absorbers, thus increasing the contrast of these lines. This effect is most visible (see Fig. 2) in the highly broadened NaI doublet $(0.59 \mu \mathrm{m})$ and KI doublet $(0.77 \mu \mathrm{m})$, the broadening also a consequence of the higher transparency of the cond cloud such that the increased pressure broadening of the lines deeper in the photosphere is seen.

The spectral changes due to a dusty cloud on a cond atmosphere at $T_{\text {eff }}=1900 \mathrm{~K}$ are of the opposite sign and on a smaller scale, but otherwise have a very similar wavelength dependence. Because, when we observe a star, we do not know a priori the phase of the variations, i.e. when the star is cloud-free, spectrophotometry alone will not distinguish between these two cases: the formation of one feature looks like the dissipation of the other. For example, the formation of a cond cloud on a dusty atmosphere will make the star get brighter in $J$ as it gets bluer in $J-K$, but this is the same signature as the dissipation 


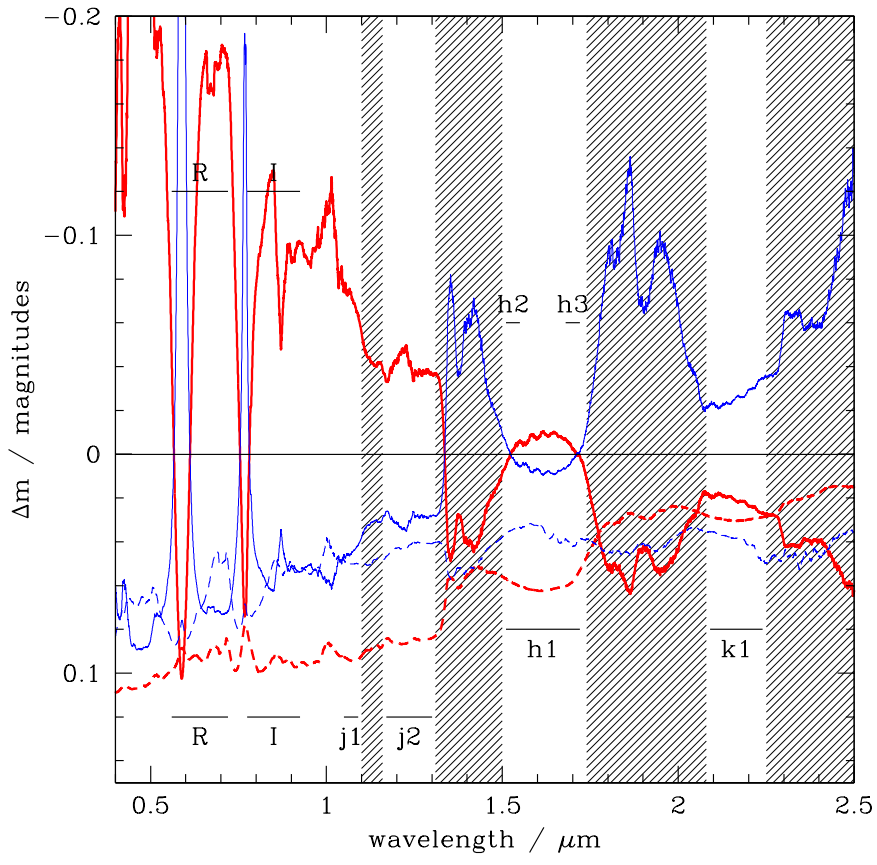

Fig. 2. The change in spectrum of an ultra cool dwarf with $T_{\text {eff }}=1900 \mathrm{~K}$ and $\log g=5.5$ due to the formation of a cloud or cool spot with a coverage factor of 0.1 according to Eq. (1). The four lines shown are for the following cases: cond cloud on a dusty atmosphere (thick/red solid line) and $200 \mathrm{~K}$ cooler spot on a dusty atmosphere (thick/red dashed line); dusty cloud on a cond atmosphere (thin/blue solid line) and $200 \mathrm{~K}$ cooler spot on a cond atmosphere (thin/blue dashed line). The cool spot is modelled using the same atmosphere type (cond or dusty) as the "background" (feature-free) atmosphere. The grey bands show regions of telluric absorption, i.e. non- or poorly accessible from ground-based observations. The horizontal labelled lines show the location of various bands defined in Table 1 . The $F W H M$ of the $R$ and $I$-bands are also shown (the latter being that used by Bailer-Jones \& Mundt 1999, 2001).

of a dusty cloud on a cond atmosphere (Fig. 2). The amplitude of the signatures differs for a given cloud coverage, but this too is unknown a priori. Only very accurate measurements could distinguish between the small differences in the wavelength dependencies for these two cases. If we could assume that surface features are rare and shortlived, the cloud-free atmosphere could be determined over time. However, the fraction of variable UCDs discovered belies this option (Sect. 1).

\subsection{Photometric signatures in flux integrated bands}

Rather than examining the variability at all resolved wavelengths in the data presented later, I have chosen to define various bands by summing the flux over continuous wavelengths regions where the variability signature is similar (Table 1 ). The bands $j 1, j 2, h 1$ and $k 1$ were chosen to avoid regions of strong telluric absorption. Variability of water absorption in the Earth's atmosphere makes reliable detection in such regions very difficult. $h 1$ and $k 1$ are essentially the conventional $H$ and $K$ bands shortened to
Table 1. Wavelength bands for analysing near infrared spectra for variability effects. The subscripts $j h$ and $h k$ are used to distinguish whether the $H$ bands were measured from the $J H$ or $H K$ spectra (see Sect. 4).

\begin{tabular}{ll}
\hline \hline band & Wavelength range $/ \mu \mathrm{m}$ \\
\hline$j 1$ & $1.05-1.09$ \\
$j 2$ & $1.17-1.30$ \\
$h 1$ & $1.51-1.72$ \\
$h 2$ & $1.51-1.55$ \\
$h 3$ & $1.68-1.72$ \\
$k 1$ & $2.09-2.24$ \\
\hline
\end{tabular}

minimize sensitivity to this. The blue end of $j 1$ was set to avoid the region $1.00-1.05 \mu \mathrm{m}$, which turned out to be very noisy in the spectra presented later in this paper. $h 2$ and $h 3$ show essentially no flux variability under cloud formation whereas they do vary under spot formation. These two bands were set specifically for a UCD with $T_{\text {eff }} \simeq 1900 \mathrm{~K}$, i.e. $2 \mathrm{M} 1145$, but as can be seen from Figs. 3 and 4 , they still have only a small signature at both higher and lower temperatures.

The most useful discriminant between a cond cloud and a cool spot on a dusty atmosphere comes from the $j 1$ and $k 1$ bands (Fig. 2). With the cloud covering $10 \%$ of the surface, the change in $j 1$ is anticorrelated with that in $k 1$, with amplitudes of 0.07 mags and 0.025 mags respectively. In contrast, with a cool spot, $j 1$ and $k 1$ are correlated with amplitudes of 0.09 mags and 0.03 mags respectively. This distinguishes between these scenarios without prior knowledge of the cloud/spot size or variability phase (i.e. the band fluxes in the absence of surface features).

Unique signatures also exist in the colours formed from these bands. These can serve to confirm a putative model from the same spectral data. Further, in some circumstances, only colour and not absolute photometric data may be available. The formation and dissipation of a cond cloud causes the colours $j 1-j 2, j 1-2, j 2-h 2$ and $h 2-k 1$ to vary (in the same direction) with amplitudes of 0.03 , 0.07, 0.04 and 0.025 mags respectively. The cool spot, on the other hand, produces variations (again in phase) in these colours of $0.005,0.035,0.03$ and 0.03 mags respectively. Although all these colour variations are in the same direction for a given model, the fact that their amplitudes differ enable us to distinguish between the models without having to know a priori the spot size. The amplitudes scale linearly for small feature coverages so the amplitude ratios permit a distinction. For the cond cloud, $j 1-j 2$, $j 1-h 2, j 2-h 2$ and $h 2-k 1$ have amplitude ratios of $3: 7: 4: 3$, whereas for the cool spots this is 1:7:6:6. The band and colour signatures are summarised in Table 2.

In order to be able to discriminate between these various scenarios at the $3 \sigma$ level for a $10 \%$ feature coverage, these amplitudes would have to be measured to an accuracy of at least 0.01 mags. In principle, these amplitudes can be used to derive the spot size, although this calls for even higher accuracy. How these required accuracies translate to individual photometric accuracies depends on 
Table 2. The predicted photometric variations (in magnitudes) due to the formation of various types of surface features covering $10 \%$ of the surface of an ultra cool dwarf with $T_{\text {eff }}=1900 \mathrm{~K}$ and $\log g=5.5$, the nominal parameters for 2M1145. The signatures are shown for the two types of atmosphere and two types of features plotted in Fig. 2: the clouds have the same effective temperature as the atmosphere; the spots are $200 \mathrm{~K}$ cooler. In the usual convention, positive signs denote decreased brightness (bands) or increased redness (colours).

\begin{tabular}{lrrrr}
\hline \hline $\begin{array}{l}\text { band or } \\
\text { colour }\end{array}$ & \multicolumn{2}{c}{ dusty atmosphere } \\
cond & \multicolumn{2}{c}{ cond atmosphere } \\
cloud & spot & dusty & cloud & $\begin{array}{r}\text { cool } \\
\text { spot }\end{array}$ \\
\hline$j 1$ & -0.070 & 0.090 & 0.045 & 0.050 \\
$j 2$ & -0.040 & 0.085 & 0.030 & 0.045 \\
$h 1$ & -0.005 & 0.060 & 0.005 & 0.035 \\
$h 2$ & 0.000 & 0.055 & 0.000 & 0.035 \\
$h 3$ & 0.000 & 0.055 & 0.000 & 0.040 \\
$k 1$ & 0.025 & 0.030 & -0.030 & 0.040 \\
$j 1-j 2$ & -0.030 & 0.005 & 0.015 & 0.005 \\
$j 1-h 2$ & -0.070 & 0.035 & 0.045 & 0.015 \\
$j 2-h 2$ & -0.040 & 0.030 & 0.030 & 0.010 \\
$h 2-k 1$ & -0.025 & 0.030 & 0.030 & -0.005 \\
\hline
\end{tabular}

the number of measurements used to determine the amplitude: the individual measurements do not need to obtain this level of photometric precision in their random errors. However, the most challenging problem will be to keep the systematic errors ${ }^{4}$ well below $1 \%$. Possible significant effects are imperfect background subtraction (especially in the NIR) and colour-dependent flux losses due to decentering of the star on the spectrograph slit (see Sects. 4 and 6). First order changes in atmospheric extinction are removed through differential (spectro)photometry, but second order colour-dependent effects may become a significant systematic error for wide bands and/or in the optical (see Bailer-Jones \& Lamm 2002 and references therein).

The wavelength regions $1.35-1.45 \mu \mathrm{m}$ and $1.80-$ $2.00 \mu \mathrm{m}$ - which are generally inaccessible from the ground due to almost total telluric absorption - would be very useful bands (Fig. 2), because they trace the variation in the dominant water absorption in UCD atmospheres. Denoting these by $t 1$ and $t 2$ respectively, we see that $j 1$ is anticorrelated with both $t 1$ and $t 2$ with amplitudes of 0.04 and 0.05 mags respectively, and $j 1-t 1$ and $t 1-t 2$ have amplitudes of 0.11 and 0.12 mags respectively, from a cond cloud on a dusty atmosphere. This is larger than the best otherwise available $j 1, k 2$ signature.

\footnotetext{
${ }^{4}$ By systematic error I mean any error which can make the measured value (e.g. the flux) correlate with some observing parameter, such as time, airmass, wavelength or position on the detector. A random error, by contrast, is one in which the measured value shows no such correlation.
}

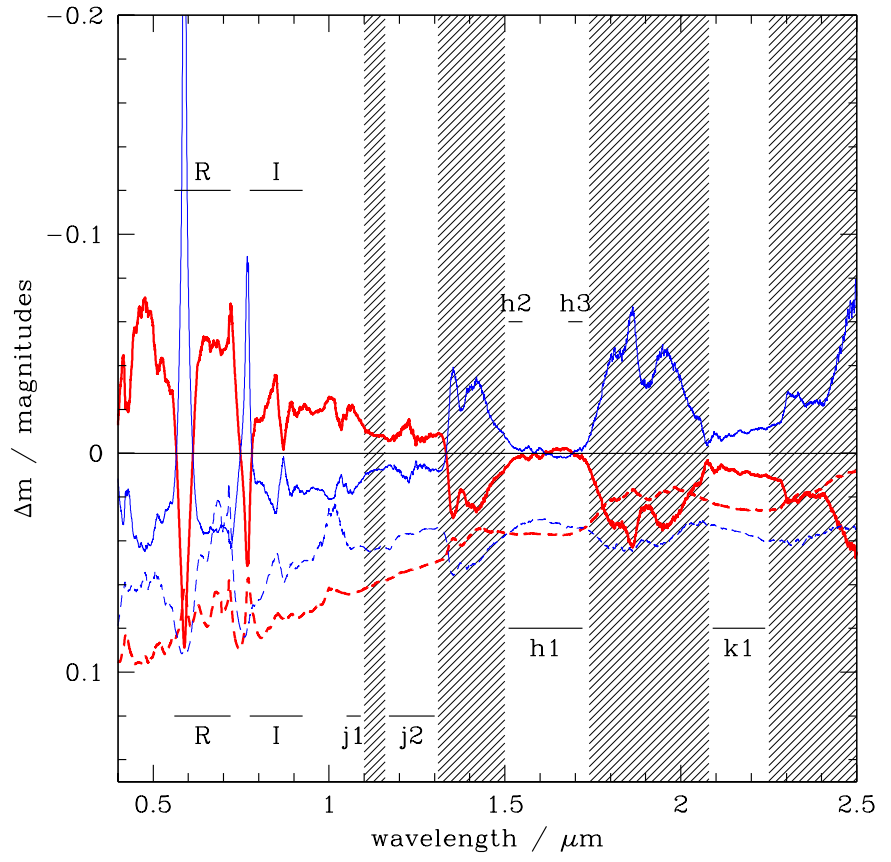

Fig. 3. Same as Fig. 2 but for a atmosphere with $T_{\text {eff }}=2100 \mathrm{~K}$.

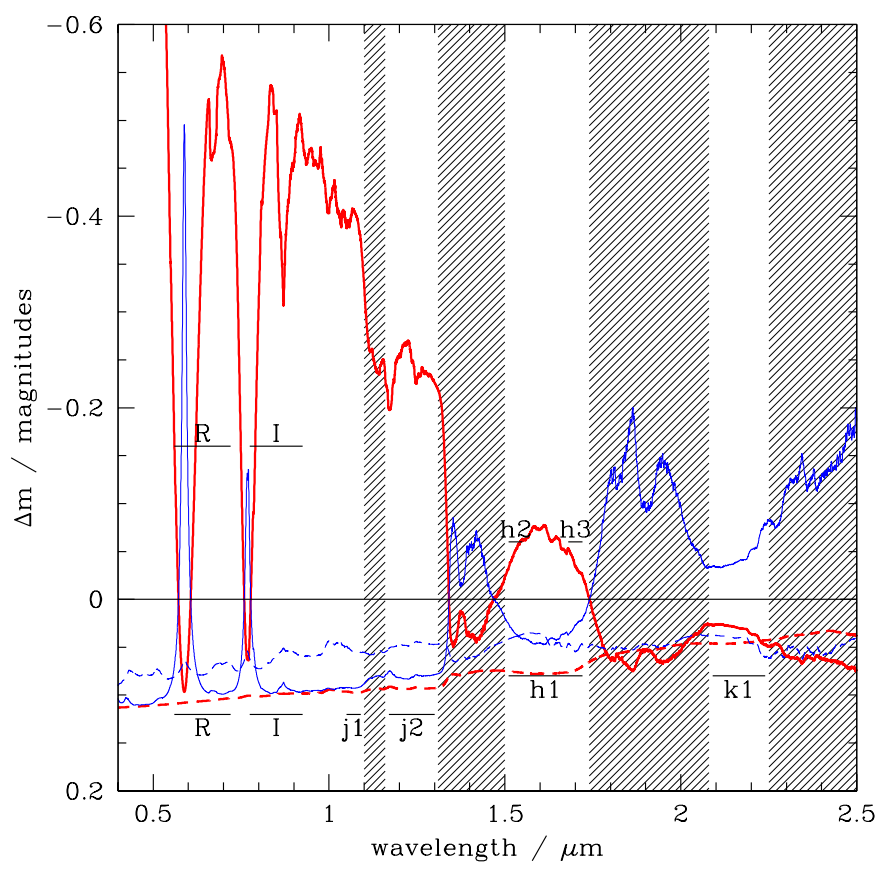

Fig. 4. Same as Fig. 2 but for a atmosphere with $T_{\text {eff }}=1700 \mathrm{~K}$. Note the different scale on the vertical axis.

\subsection{Spot and cloud signatures at $1700 \mathrm{~K}$ and $2100 \mathrm{~K}$}

The above discussion is relevant for a UCD with an effective temperature of $1900 \mathrm{~K}$ and $\log g=5.5$. From the plots in Allard et al. (2001), it appears that a different surface gravity will make no detectable difference to the variability amplitudes discussed. However, increasing or decreasing the effective temperature by $200 \mathrm{~K}$ has a significant effect, as can be seen in Figs. 3 and 4 . 
At $2100 \mathrm{~K}$, the cloud variability amplitude is reduced because of the smaller differences between the opacity sources in the dusty and cond models at higher temperatures, particularly blueward of $1.3 \mu \mathrm{m}$. This is simply because less dust condenses at higher temperatures, so it makes less difference whether that stays in suspension in the atmosphere or is precipitated out. The differences are enhanced at the lower effective temperature $(1700 \mathrm{~K})$ for the converse reason, i.e. more condensation of gas-phase species (and their precipitation in the cond models).

The same bands as described above for $1900 \mathrm{~K}$ can be used at $2100 \mathrm{~K}$, but now the cloud signatures are reduced by a factor of four in the $j 1$ and $j 2$ bands. For a $10 \%$ cloud coverage, this is probably below the level at which systematic errors can be controlled from ground-based observations. However, at $2100 \mathrm{~K}$, the amplitude of the cool spot signature is still relatively large, and amenable to detection.

At $1700 \mathrm{~K}$, the cloud signatures are greatly enhanced and are much larger than the cool spot signatures, such that the $j 1-h 1$ colour has an amplitude of 0.4 mags due to a cloud and only 0.02 mags due to a cool spot (both covering $10 \%$ of the surface). However, it must be recalled that below $1800 \mathrm{~K}$ UCDs are generally better fit by cond atmospheres, and dusty clouds on such atmospheres show a much weaker signature at $1700 \mathrm{~K}$ (Fig. 4). Moreover, the cloud signature is probably sensitive to the degree of dust precipitation at these intermediate temperatures and this is not accounted for in the present models (see Sects. 1 and 3.1).

\subsection{Signature in the optical and mid infrared regions}

I have focused on signatures in the NIR region as that is the region observed and analysed in the rest of this paper. However, there are strong signatures in the optical spectrum, as can be seen in Figs. 2-4. There is a trade-off here, of course, because ultra cool dwarfs are much fainter in the optical. The region $0.8-1.0 \mu \mathrm{m}$ behaves in a similar fashion to the $j 1$ band and the region $0.65-0.72 \mu \mathrm{m}$ shows an even stronger signature across a range of effective temperatures. Very narrow bands centered on the NaI $(0.59 \mu \mathrm{m})$ and $\mathrm{KI}(0.77 \mu \mathrm{m})$ atomic absorption lines are in principle a good discriminant between clouds and cool spots when combined with a band in the range $0.8-1.1 \mu \mathrm{m}$, but the narrowness required $(\sim 0.02 \mu \mathrm{m})$ to avoid the opposite signature in the line wings may preclude their use from a signal-to-noise ratio $(S N R)$ point of view.

The mid infrared appears to contain no wavelength regions which are of particular advantage over the near infrared for distinguishing cool spots from dust-related clouds, at least not at around $1900 \mathrm{~K}$ (Fig. 5). However, at lower temperatures methane forms, and there remains the interesting possibility of looking for methane clouds either in the near or mid infrared (Bailer-Jones, in preparation).

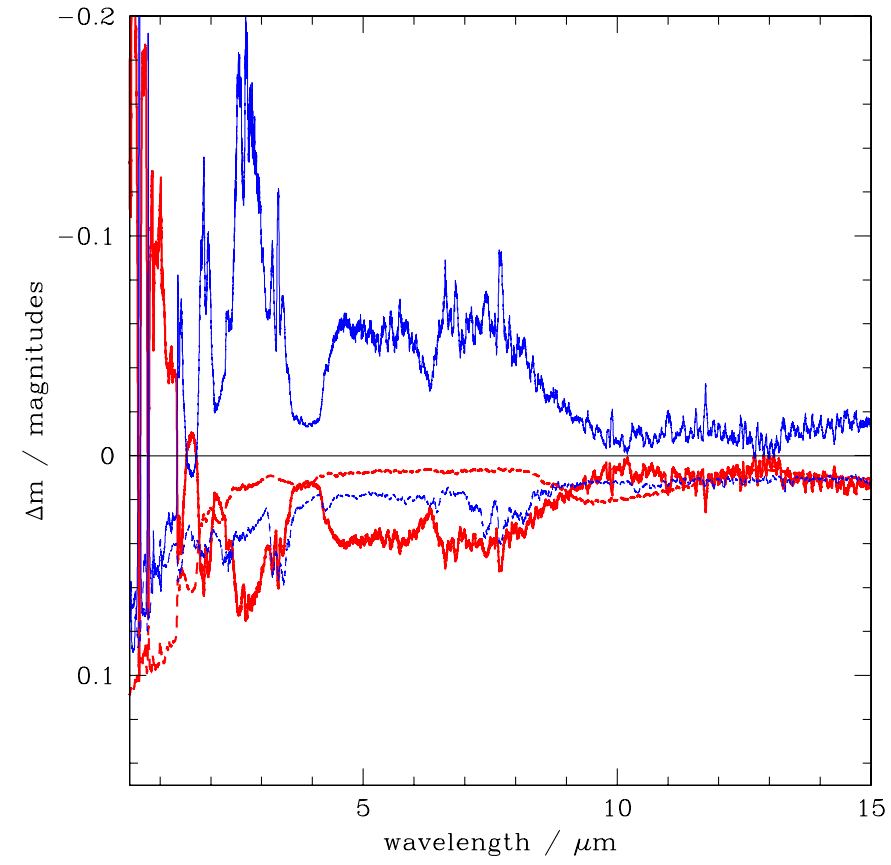

Fig. 5. Same as Fig. 2 but for the mid infrared wavelength region.

\section{Data acquisition}

The analyses of the previous section were motivated by the desire to interpret infrared spectra of the L1.5 dwarf 2M1145. Spectra were obtained using the Omega Cass infrared imager/spectrograph mounted on the Cassegrain focus of the $3.5 \mathrm{~m}$ telescope at Calar Alto, Spain. The detector is a $1024 \times 1024 \mathrm{HgCdTe}$ HAWAII- 1 focal plane array sensitive between 0.8 and $2.5 \mu \mathrm{m}$. The low resolution camera was used, giving an image scale of $0.3^{\prime \prime} /$ pix. The resolution $420(=\lambda / \Delta \lambda)$ grism was used in combination with one of two filters: with a $J H$ filter it provided the wavelength range $0.98-1.84 \mu \mathrm{m}$ unoverlapped in first order at a dispersion of $0.0021 \mu \mathrm{m} / \mathrm{pix}$ (plus a second order which was not used); with an $H K$ filter the wavelength range $1.48-2.41 \mu \mathrm{m}$ was obtained in first order at the same dispersion. The slit had a width of $0.9^{\prime \prime}$ and length of $141^{\prime \prime}$.

The instrument was oriented such that the spectrograph slit was at an angle $10.8^{\circ}$ East of North, to include both $2 \mathrm{M} 1145$ and the bright reference star $79^{\prime \prime}$ to the South (see Sect. 2). The detector edges remained parallel to the slit. The observing procedure was as follows. Using a direct image, the stars are brought to the approximate required position on the detector. A guide star is acquired and the slit brought into the beam at the exact position of the stars. Then the grism is inserted and several sequences of observations made. One sequence is defined as two exposures $(1,2)$ at the same telescope position, followed by a telescope offset of $20^{\prime \prime}$ along the slit and another two exposures $(3,4)$. The same sequence was then normally repeated with the $H K$ filter. This was continued for between three and seven sequences in each filter until it was noticed that the counts in the stars were decreasing due to a decentering of the slit with respect to 
Table 3. Observing log of spectrophotometric observations of $2 \mathrm{M} 1145$ in terms of the number of cycles and sequences in $\mathrm{JH}$ and $H K$ (see text for definition of these terms). Night 1 started near the end of 29 February 2000 UT at around JD 2451604.4.

\begin{tabular}{cccc}
\hline \hline Night & Cycle & $\begin{array}{c}\text { No. of } J H \\
\text { sequences }\end{array}$ & $\begin{array}{c}\text { No. of } H K \\
\text { sequences }\end{array}$ \\
\hline 1 & 1 & 6 & 6 \\
& 2 & 5 & 4 \\
& 3 & 7 & 6 \\
2 & 4 & 5 & 4 \\
& 1 & 7 & 7 \\
& 2 & 5 & 5 \\
3 & 3 & 3 & 3 \\
3 & 4 & 7 & 6 \\
& 1 & 5 & 5 \\
\hline
\end{tabular}

the stars (which ranged from $0.6^{\prime \prime}$ to $1.2^{\prime \prime}$ over two hours, despite the guiding). Then, without moving the telescope, grism or slit, the telescope dome was moved across the field and a series of dome flat fields obtained. Such a set of sequences constitutes a cycle. This procedure was adopted in order to obtain flat fields with as similar an optical path to the stars as possible and to keep this path as constant as possible through minimal movement of the optical elements. Each exposure used an integration time of $2 \mathrm{~min}$. The readout time is essentially zero, so a single sequence took just over $8 \mathrm{~min}$.

Data were obtained on three consecutive nights starting on 29 February 2000 (Table 3 ). The seeing in $J H$ was usually $1.0^{\prime \prime}-1.4^{\prime \prime}$ but occasionally as high as $2.0^{\prime \prime}$. The weather was generally very clear, but occasionally thin cirrus was present.

\section{Image processing and spectral extraction}

The data reduction and spectral extraction was mostly performed using IRAF ${ }^{5}$. Careful processing is required to avoid biasing the data, so the procedure developed is described. The $J H$ and $H K$ spectra were treated separately.

From the four images $(1,2,3,4)$ in a sequence, four difference images were produced: $a=1-3, b=-a, c=2-4$, $d=-c$. This removes most of the sky background plus the detector bias pattern. These images were then flat fielded using the end-of-cycle dome flats described in Sect. 4. These flats show absorption features due to the air in the circa $50 \mathrm{~m}$ optical path between lamp and detector. However, as the dispersion function for the flats and the science images is the same, this provides a valid flatfielding of the detector in the spatial direction to put the two dither positions ( $\mathrm{a}, \mathrm{c}$ and $\mathrm{b}, \mathrm{d}$ ) on the same photometric scale. Small shifts of the slit with respect to the detector in the wavelength direction change the flat field value at each wavelength, but as this is the same change for both $2 \mathrm{M} 1145$ and the reference star, it is not detrimental

\footnotetext{
${ }^{5}$ the Image Reduction and Analysis Facility, provided by the National Optical Astronomy Observatories.
}

to spectrophotometry in the relative spectra (that is, the ratio of these - see below). Bad pixels (as identified from dark images) were removed from the flat-fielded difference images by linearly interpolating over them in the spatial direction.

These four images provide four independent spectra of each of the target and reference stars. The spectra were extracted using the one-dimensional optimal extraction technique implemented in IRAF's "apsum" task. As these images are sky subtracted, pixel cleaning (e.g. to remove cosmic rays) could not be used reliably within this package (but the effect of cosmic rays was found to be minimal). A local sky subtraction at each wavelength using the median value of regions on each side of the spectrum was found to be necessary to further reduce the sky contribution. (The image differencing does not remove changes in the sky background occuring over the four minutes elapsing between images 1 and 3 or 2 and 4 ).

The spectra were wavelength calibrated using 15-25 OH airglow lines identified in Oliva \& Origlia (1992) and present in spectra extracted from the undifferenced images. A quadratic dispersion function was most suitable, giving RMS errors in the fits of less than $6 \mathrm{e}^{-4} \mu \mathrm{m}$ $(<0.3$ pixels $)$. The spectra were then transformed to a common linear wavelength scale with $0.0025 \mu \mathrm{m}$ wide bins using cubic spline interpolation. These spectra of the individual objects shall be referred to as the nominal spectra. Theoretical error spectra were calculated from these based on the Poisson noise from star and sky and read noise contributions in the weighted extraction aperture.

Relative spectra are produced by dividing the target nominal spectrum by the reference nominal spectrum from the same difference image. On the assumption that the reference star is photometrically stable, this should remove any time-dependent Earth atmosphere transmission variations from a time series of observations. As the relative photometry is in a series of narrow wavelength bins $(0.0025 \mu \mathrm{m})$, second order colour-dependent extinction effects are negligible. The four relative spectra from a sequence are then averaged to create a higher $S N R$ combined spectrum. This is shown in Figs. 6 and 7 along with the combined nominal spectra. Some features are recognisable, such as the NaI line at $1.14 \mu \mathrm{m}$ (at the bottom of a telluric water absorption band), $\mathrm{FeH}$ at $1.20 \mu \mathrm{m}$ and the resolved KI doublet at $1.25 \mu \mathrm{m}$.

A band magnitude, $m$ (see Sect. 3.3) is calculated by

$m=-2.5 \log _{10}\left(\frac{1}{4} \sum_{i=1}^{i=4} f_{i}\right)$

where $f_{i}$ is the sum of the (relative) flux over the wavelength interval for that band (Table 1 ) for the $i$ th (relative) spectrum in that cycle (see Sect. 4). The idea is always to first ratio the target measurement with the reference measurement taken simultaneously and then average, thus removing concurrent fluctuations in the Earth's atmospheric transmission. The same procedure is used for colours by replacing $f_{i}$ in Eq. (2) with the ratio of fluxes obtained 

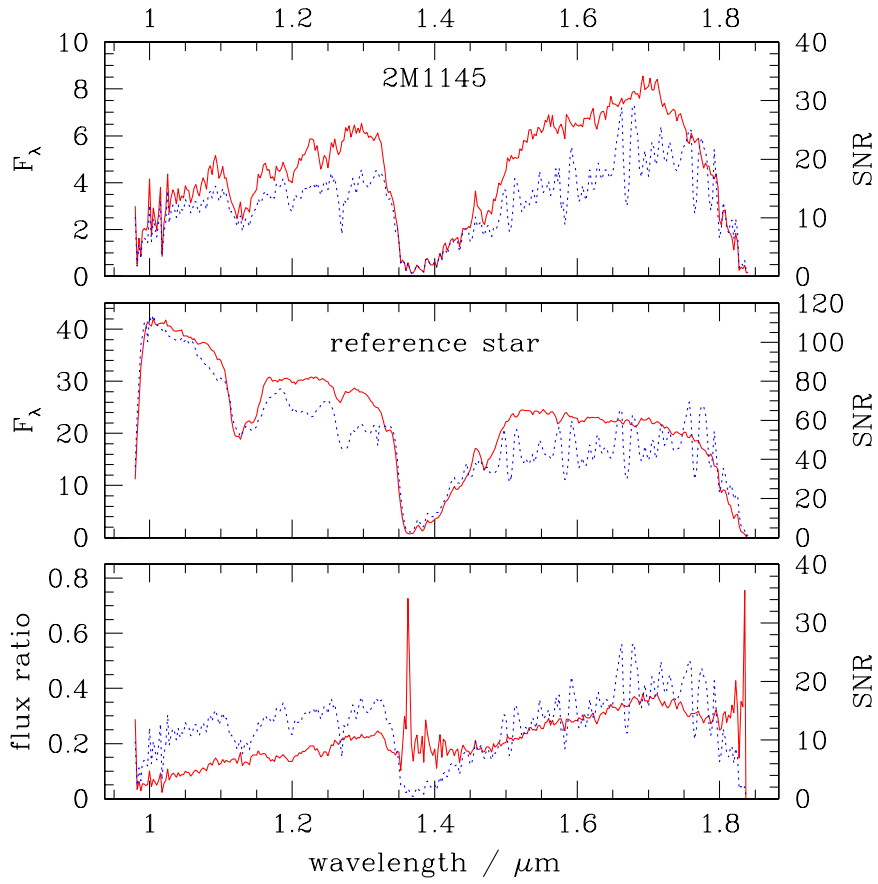

Fig. 6. Example spectra obtained in the $J H$ filter. The top two panels show the nominal spectra of $2 \mathrm{M} 1145$ and the reference star and the bottom panel shows the relative spectrum (solid lines, left axis). The corresponding signal-to-noise ratios $(S N R \mathrm{~s})$ are also shown (dotted lines, right axis). The nominal spectra shown are the averages of four consecutive two minute exposures (i.e. from one sequence). The spectra of the two objects are obtained simultaneously using a long slit. A relative spectrum is formed by taking the ratio of these two spectra. The combined relative spectrum shown is the average of these four relative spectra (which is not simply the ratio of the two nominal spectra shown). The spectra have been flat fielded and sky subtracted, but not corrected for telluric absorption. Of course, many telluric features are absent from the relative spectrum, as intended. The flux scales for the two nominal spectra are the same and in arbitrary units. The spectra are from the fifth sequence on night 1 . The low signal and $S N R$ between 1.33 and $1.45 \mu \mathrm{m}$ is the familiar opaque region of the Earth's atmosphere between the $H$ and $K$ bands.

from a (relative) spectrum. Colours formed in this way from relative spectra are called relative colours. As I only ever look at changes in band magnitudes and colour indices, the zero point is irrelevant. Note that the $j 1$ and $j 2$ bands cannot be obtained from the $H K$ data and the $k 1$ band cannot be obtained from the $J H$ data, so colour indices between these cannot be formed from contemporaneous data. The bands $h 1, h 2$ and $h 3$ can be formed from either the $J H$ or $H K$ spectrum, which I will distinguish where necessary using subscripts, e.g. $h 1_{j h}$ and $h 1_{h k}$.

Photometric errors are calculated for the bands by propagation of the errors in the nominal spectra. The validity of these theoretical errors has been assessed by comparison to empirical errors obtained from the standard error in the mean of the four relative spectra, and it is found that they generally agree with a few tens of percent. These empirical errors show some large fluctuations, probably

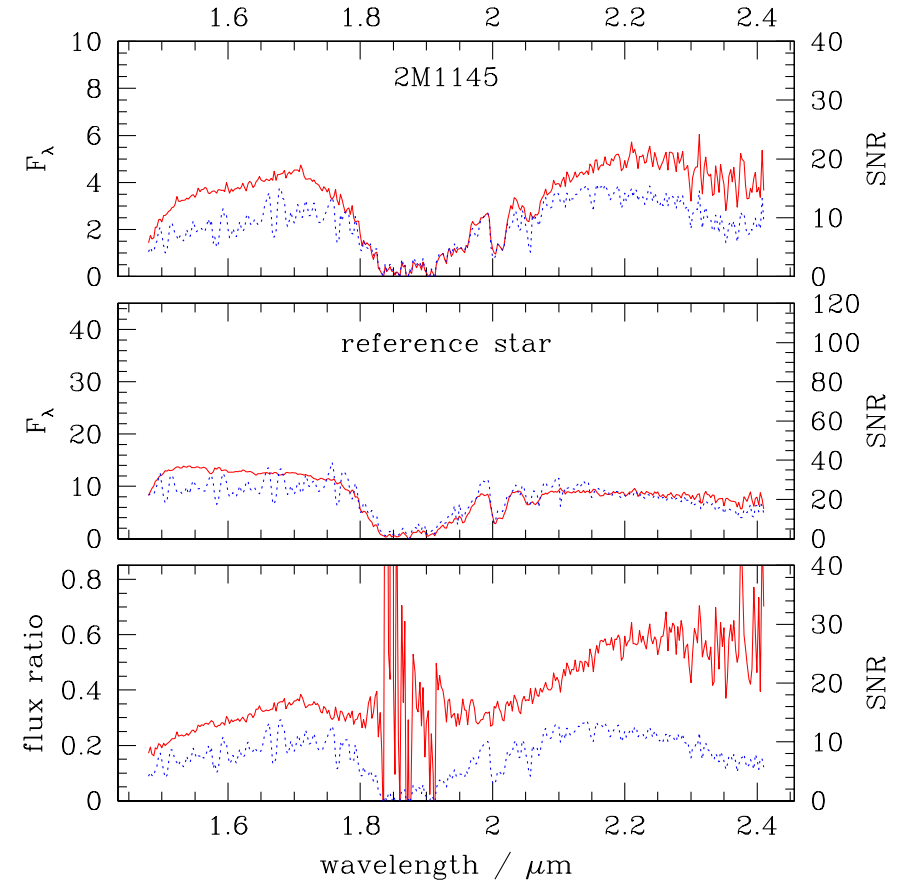

Fig. 7. Same as Fig. 6 but for the $H K$ filter. The spectra are from the fifth sequence on night 1 . The flux is in the same (arbitrary) units as in Fig. 6. The low signal and $S N R$ between 1.80 and $1.95 \mu \mathrm{m}$ is due to strong water absorption by the Earth's atmosphere.

because they are obtained from the scatter between just four spectra. For this reason I use the theoretical errors throughout.

\section{Differential variability analysis}

The first analysis was to see whether differential spectrophotometry in the relative spectra is reliable. Figure 8 shows this for the three bands $j 1, h 2_{j h}$ and $k 1$. All three show a series of correlated decreases in flux. The gaps between the successive trends correspond to the transitions between observing cycles (Sect. 4). This effect is caused by the decentering of the star on the spectrograph slit during a cycle. To affect the relative spectra, the degree of decentering must be different for the two objects, either because the slit did not remain parallel to the line joining the stars, or because the slit edges were not sufficiently parallel to each other. Although such decentering was expected (from differential flexing between the guide camera and the instrument), it was not expected to be as large as 0.2 mags per hour. Thus we cannot perform pure differential spectrophotometry with these data.

Colour variability within 2M1145 alone also turns out to be affected by the same problem. Figure 9 shows the variation in the colours $j 1-h 2$ and $h 2-k 1$ formed from the nominal (rather than relative) spectra of 2M1145 and the reference star. We immediately see that within each cycle both objects appear to get bluer, i.e. more red light than blue light is lost from the slit decentering. This is most likely due to atmospheric refraction, such that the 


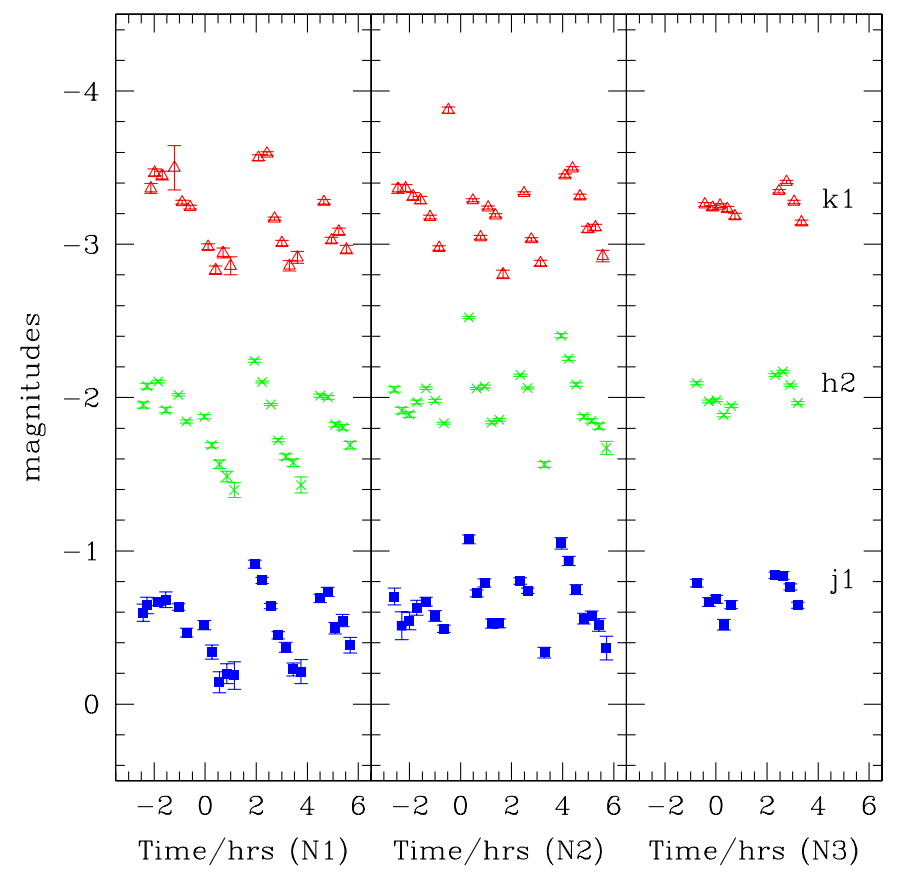

Fig. 8. Band magnitudes for the relative spectra of $2 \mathrm{M} 1145$ (i.e. spectrum of $2 \mathrm{M} 1145$ divided by that of the reference star) for $j 1$ (solid square), $h 2_{j h}$ (cross) and $k 1$ (open triangle). The horizontal axis in each case is the time in hours from UT0 on nights 1, 2 and 3 (left to right). Each band has an arbitrary offset on the vertical axis which is the same for all three nights. The bands are defined in Table 1.

redder part of the refracted image is that which is more decentered with respect to the slit.

In spite of these problems, we find that colour variability in the relative spectra is reliable, in the sense that there is no systematic trend within a cycle. This can be seen in Fig. 10 for the colours $j 1-j 2, j 1-h 2, j 2-h 2$ and $h 2-k 1$. That the colours formed from the relative spectra can be reliable despite the problems seen in Figs. 8 and 9 can be understood from the fact that both 2M1145 and the reference star show the same degree of "bluening" as a result of the slit decentering (see Fig. 9).

It is fairly clear from a visual inspection of Fig. 10 that none of the four light curves shows good evidence for variability. This can be put on a quantitative basis using a statistical test, for example the $\chi^{2}$ test (see Sect. 4.1 of BJM). The probabilities that there is a real variability (i.e. not related to the noise) are shown in Table 4 . This table also gives the amplitudes of the relative colour light curves. It is tempting to study the ratios of these amplitudes and compare them with the predictions given in Sect. 3. However, the dominant contribution to these amplitudes is noise, so we would first have to statistically subtract the noise contribution. This can only be done once we have a model for both the noise and the underlying variability. Taking the former to be Gaussian with standard deviation $\sigma_{\text {noise }}$ and the latter to be a sinusoidal signal, then the rms amplitude of the underlying signal, $\sigma_{\mathrm{var}}(=0.5 \sqrt{\pi}$ times the

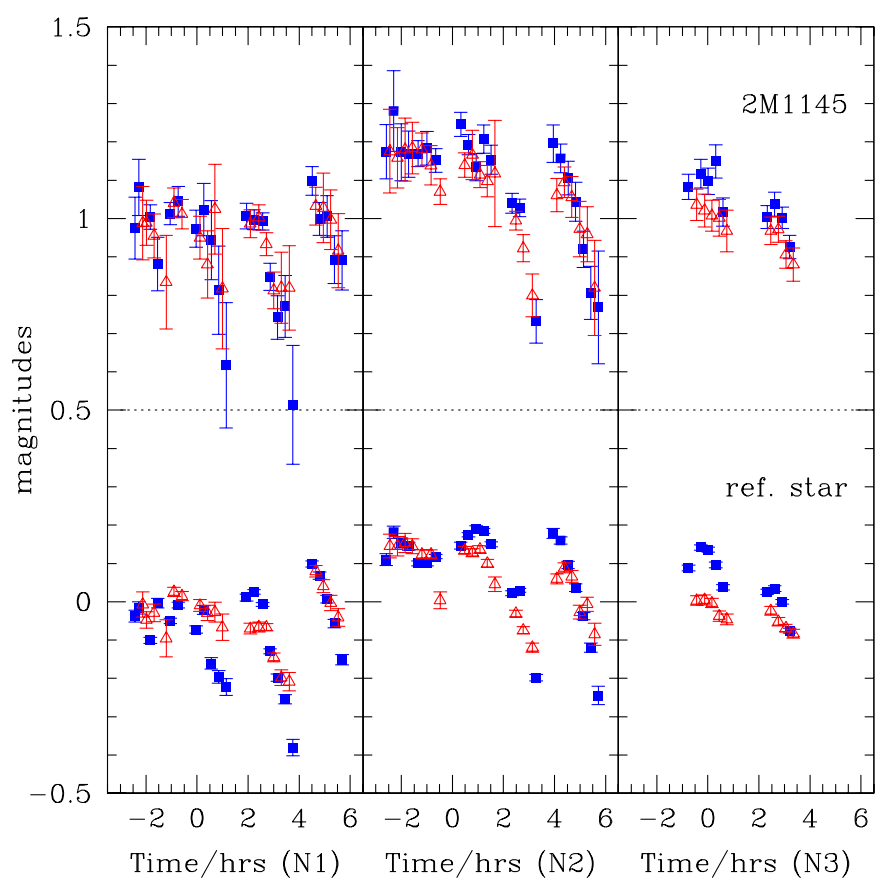

Fig. 9. Colour variability in the nominal (not relative) spectra of $2 \mathrm{M} 1145$ and the reference star in the colours $j 1-h 2$ (filled squares) and $h 2-k 1$ (open triangles). The colours have been set to a mean (across all nights) of 0.0 for 2M1145 and 1.0 for the reference star. Points lying above the dashed line are for $2 \mathrm{M} 1145$, those below are for the reference star. The time axis is the same as in Fig. 8.

Table 4. Amplitudes of the variations in the four relative colour light curves shown in Fig. 10. Two measures of light curve amplitude are given: $\sigma_{\mathrm{m}}$, the rms (root-mean-square) of the relative magnitudes, and $\overline{\left|m_{\mathrm{d}}\right|}$, the average modulus deviation in the light curve from its mean value (the linear equivalent of an RMS). $\sigma_{\text {noise }}$ is the median of the theoretical errors (the error bars shown in Fig. 10). $1-p$ is the probability calculated from the $\chi^{2}$ test that the variability across all points is "real", i.e. not due to the empirical photometric errors.

\begin{tabular}{lrrrr}
\hline \hline Colour & $\begin{array}{r}\sigma_{\mathrm{m}} \\
\text { mags }\end{array}$ & $\begin{array}{r}\overline{\left|m_{\mathrm{d}}\right|} \\
\text { mags }\end{array}$ & $\begin{array}{c}\sigma_{\text {noise }} \\
\text { mags }\end{array}$ & $1-p$ \\
\hline$j 1-j 2$ & 0.044 & 0.033 & 0.034 & 0.41 \\
$j 1-h 2$ & 0.055 & 0.040 & 0.044 & 0.73 \\
$j 2-h 2$ & 0.032 & 0.026 & 0.032 & 0.18 \\
$h 2-k 1$ & 0.040 & 0.031 & 0.054 & 0.00 \\
\hline
\end{tabular}

peak-to-peak amplitude), is ${ }^{6} \sqrt{\sigma_{m}^{2}-\sigma_{\text {noise }}^{2}}$. However, as we have no evidence for significant variability in any single colour, this is not appropriate for the present data set.

The light curves in Fig. 10 are the same (within the errors) if $h 1$ or $h 3$ is used instead of $h 2$. This can be see in

\footnotetext{
6 This can only be a rough approximation, however, as we do not expect features which evolve faster than a rotation period to necessarily show a sinusoidal signature. There is also the issue of how to form a suitable single value of $\sigma_{\text {noise }}$ given the large variation in the errors in Fig. 10.
} 


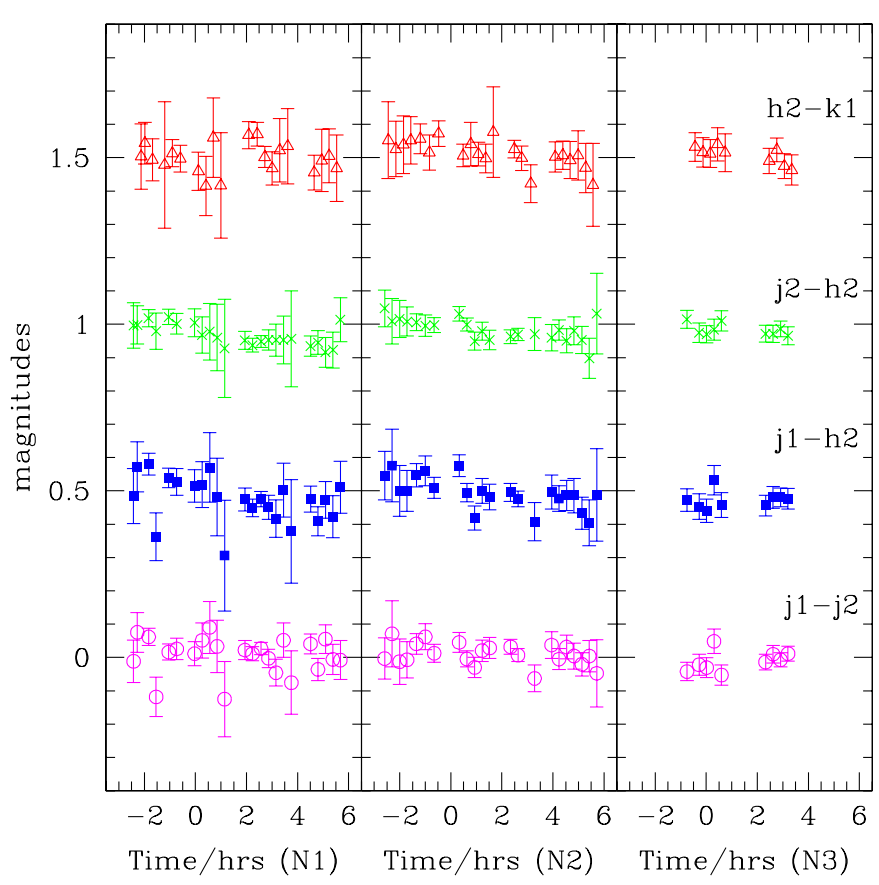

Fig. 10. Colours formed from the relative spectra (2M1145 divided by the reference star). The colours shown are, from bottom to top, $j 1-j 2$ (open circles), $j 1-h 2$ (filled squares), $j 2-h 2$ (crosses) and $h 2-k 1$ (open triangles). Each colour has an arbitrary offset on the vertical axis which is the same for all three nights. A more positive magnitude means that 2M1145 gets redder relative to the reference star. The time axis is the same as in Fig. 8. Such light curves can, in principle, be used to distinguish between variability due to spots and dust-related clouds.

Fig. 11, where the colours $j 2-h 1, j 2-h 2$ and $j 2-h 3$ have virtually the same light curves.

The $\chi^{2}$ test does not take into account any correlations between the colour variations, which is what the observing procedure was designed to exploit. Figure 12 shows that there is in fact a correlation in the relative colour variability for the colours $j 1-j 2$ and $j 1-h 2$. Despite the large errors, there is a significant positive correlation: the correlation coefficients for nights 1,2 and 3 are $0.89,0.71$ and 0.81 respectively, and 0.83 for the whole data set together. Random variations due to noise are very unlikely to give such large correlations. There is also no trend of increasing or decreasing colour within a night or an observing cycle (Fig. 10), so no evidence that this colour correlation is telluric in origin or an observational artifact. Thus while the noise on individual measurements is too large to measure a significant amplitude in a single colour, the colour variations are positively correlated and indicate something intrinsic to the object observed (either $2 \mathrm{M} 1145$ or the reference star). In contrast, Fig. 13 shows that $j 2-h 2$ has a negligible correlation with $j 1-j 2$ (the correlation coefficients for nights 1,2 and 3 are $0.20,0.04$ and 0.52 respectively, and 0.08 for all nights together).

As discussed in Sect. 3, a positive correlation between any of these colours alone in insufficient to distinguish between cond clouds and cool spots on a dusty atmosphere.

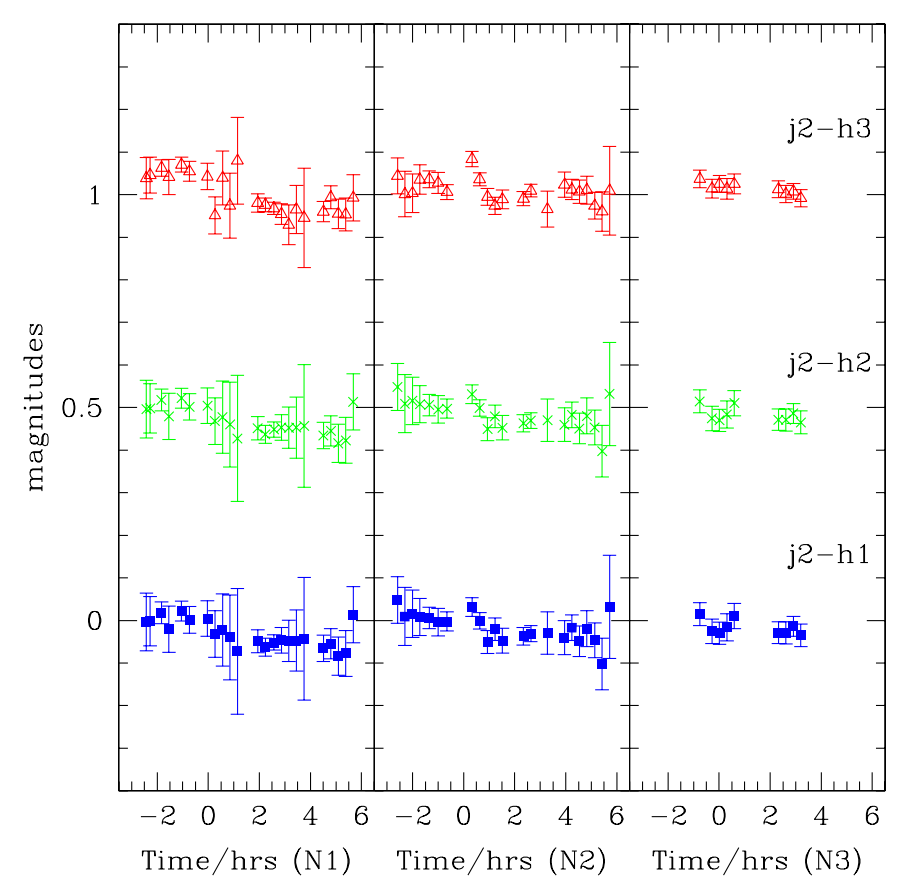

Fig. 11. As for Fig. 10 but for the relative colours (from bottom to top) $j 2-h 1$ (filled squares), $j 2-h 2$ (crosses) and $j 2-h 3$ (open triangles). This figure demonstrates that the relative colour light curves are essentially identical for all three $H$ bands defined in Table 1.

However, for a $200 \mathrm{~K}$ cooler spot on a dusty atmosphere at $1900 \mathrm{~K}$ or lower, there is very little variation in $j 1-j 2$ compared to $j 1-h 2$ or $j 2-h 2$. Therefore, in the presence of noise, we would expect both $j 1-h 2$ and $j 2-h 2$ to be equally uncorrelated with $j 1-j 2$ for such a spot. We find that the former is correlated whereas the latter is not. This is inconsistent with this particular cool spot model. For a cond cloud we would expect both to be correlated, but in the presence of noise we would observe a larger correlation between $j 1-h 2$ and $j 1-j 2$ than between $j 2-h 2$ and $j 1-j 2$ simply because the amplitude is larger. This difference becomes more pronounced at lower temperatures. Thus the observed correlations are more consistent with a cond cloud than a cool spot, for a dusty atmosphere at 1900 K. Note, however, that a cool spot on a hotter dusty atmosphere $(2100 \mathrm{~K}$, see Fig. 3 ) would produce a similar effect to that seen. Thus until we can measure amplitudes more precisely (through higher precision observations and an appropriate statistical subtraction of the errors), the interpretation of this colour correlation should be treated with some caution.

The data in the present paper were obtained quasisimultaneously with the $I$-band data reported in BJM. The latter are reproduced in Fig. 14 for the overlapping time interval (correcting for the axis errors in BJM reported in the erratum). Alongside this is shown the variability in two relative colours from the present data set. On night 1 , there is reasonable evidence to suggest that as 2M1145 became brighter in $I$ it also became bluer in 


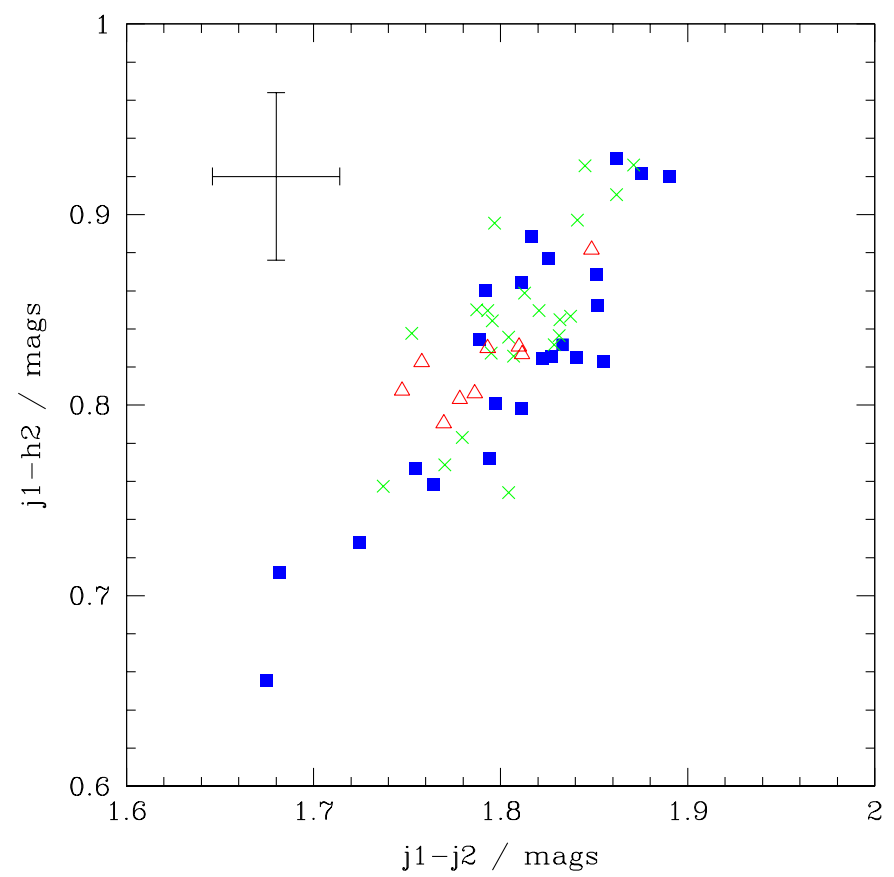

Fig. 12. Correlation between the two relative colours $j 1-j 2$ and $j 1-h 2$. The different symbols refer to observations on the different nights: night 1 (solid squares); night 2 (crosses); night 3 (open triangles). The median error is shown in the top-left corner.

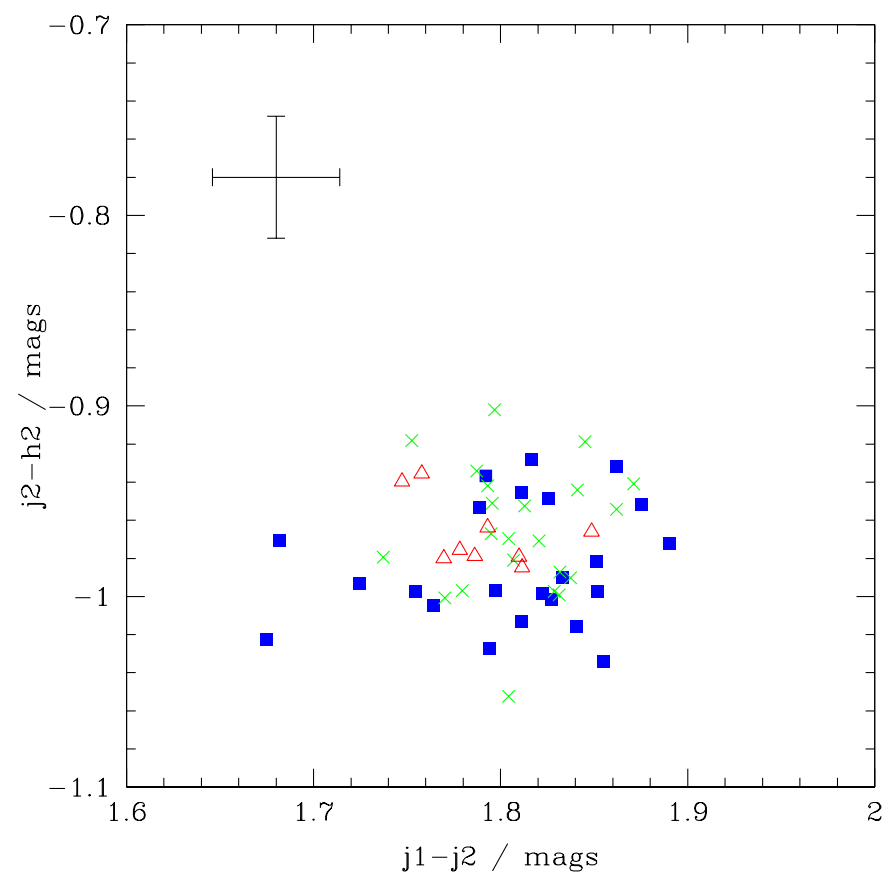

Fig. 13. Same as Fig. 12 but for the colours $j 1-j 2$ and $j 2-h 2$.

$j 1-h 1$. Both changes are significant according to the $\chi^{2}$ test $(1-p=0.99$ for $I, 1-p>0.99$ for $j 1-h 1)$. This is still significant if $h 1$ is replaced with $h 3$, but not so significant $(1-p=0.90)$ for $j 1-h 2$. This is the signature expected for the appearance of a cond cloud on a dusty atmosphere or the dissipation of a cool spot on the same atmosphere (Fig. 2).

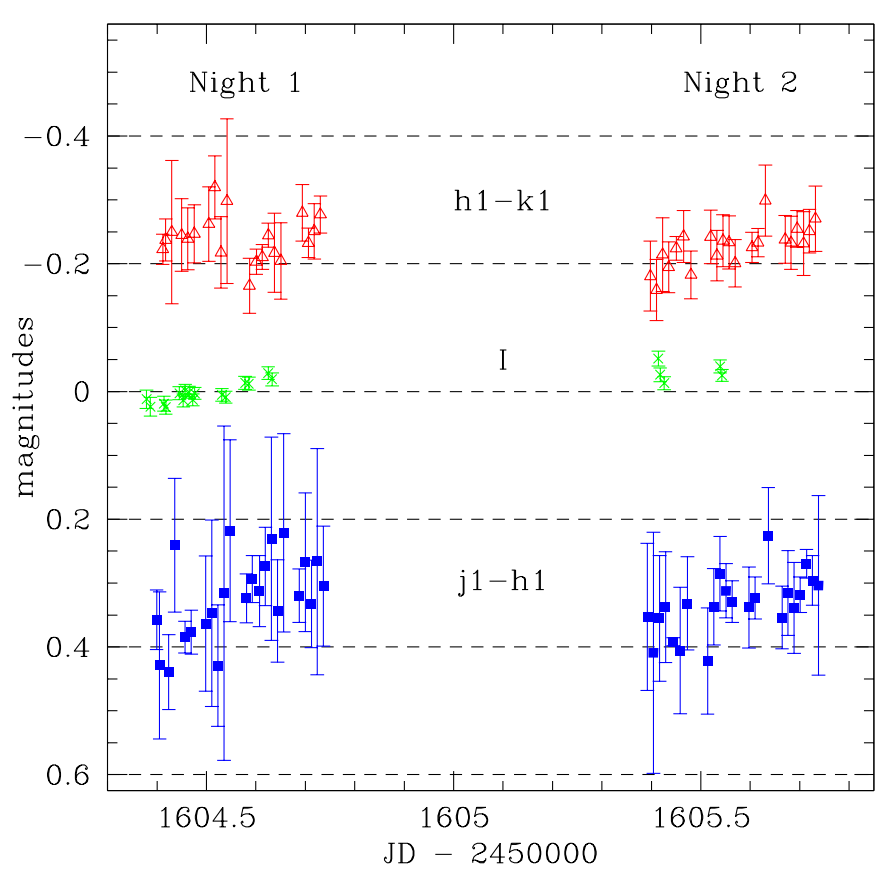

Fig. 14. Variation in $2 \mathrm{M} 1145$ of the relative colours $j 1-h 1$ (filled squares) and $h 1-k 1$ (open triangles) plotted along with the differential I-band photometry of 2M1145 (crosses) obtained simultaneously on the $2.2 \mathrm{~m}$ telescope at the same site (latter taken from Fig. 3 of BJM). Moving up the page the $I$-band gets brighter and the colours bluer. The night labels refer to the present data set (Table 3). Note that Fig. 3 of BJM is plotted the other way up (see that paper's erratum).

\section{Summary and discussion}

To summarise the results of the analysis for 2M1145: a formal $\chi^{2}$ test does not reveal any variability at the $99 \%$ confidence level across the whole data set in any of the four relative colour indices given in Table 4. Relative colours means the ratio between two band fluxes, each formed by summing flux in a relative spectrum (2M1145 divided by reference) over a specific wavelength region (see Sect. 5). Relative photometry and absolute colours (i.e. the colour of 2M1145 alone) could not be used due to time-dependent light losses from the slit. The relative colours appear unaffected by this and there is no reason to consider them as unreliable. Although no single colour shows significant variation (across the whole observing period), there is a significant correlation between the $j 1-j 2$ and $j 1-h 2$ relative colours. This is not expected from random noise and does not appear to be caused by the slit losses. I therefore conclude it to be intrinsic to either $2 \mathrm{M} 1145$ or the reference star. The reference star is most likely to be mid or late-type $\mathrm{F}$ dwarf and not a short period variable (see Sect. 2) so the variability is probably not in this object. Assuming that the variability is intrinsic to $2 \mathrm{M} 1145$, then it is more consistent with dust-free (cond) clouds rather than a dusty cool spot, assuming 2M1145 to have an overall dusty atmosphere and an effective temperature of $1900 \mathrm{~K}$. However, if 2M1145 is actually hotter $(2100 \mathrm{~K})$, then cool spots are equally consistent with these 
data. It was found that the contemporaneous $I$-band data of 2M1145 from BJM show a slight correlation with the present data in the sense that 2M1145 became brighter in $I$ as it became bluer in $j 1-h 1$. Although the data were obtained at the same site, BJM have argued that the $I$-band variability is not due to changes in the Earth's atmosphere, so we can consider this correlation to be real. It is consistent with either a cool spot or a cond cloud on a dusty atmosphere.

The present data allow upper limits to be placed on the size of surface features based on what peak-to-peak amplitude would have been required to produce a significant $\chi^{2}(1-p>0.99)$. Assuming a constant noise RMS of $\sigma_{\text {noise }}$, variability could be detected in a relative colour if the underlying (i.e. noise-free) peak-to-peak amplitude were greater than about $1.4 \sigma_{\text {noise }}$. Using the median noise in each relative colour from Table 4 , this can be compared with the signatures of the spots described in Sect. 3 .

If $2 \mathrm{M} 1145$ has a dusty atmosphere and an effective temperature of $1900 \mathrm{~K}$, then the data require that any coherent cond cloud features cover less than 10-15\% of the projected surface. Alternatively, cool spots (with $\Delta T=200 \mathrm{~K}$ ) must cover less than $20 \%$ of the surface. Even cooler spots would be restricted to a smaller coverage. If a cond atmosphere is assumed instead of a dusty one, then dusty clouds on this have essentially the same signature as cond clouds on the dusty atmosphere (see Fig. 2). Cool spots, on the other hand, have a weaker signature and could cover up to about $40 \%$ of the surface before being detected.

If $2 \mathrm{M} 1145$ is warmer (with a dusty atmosphere), then clouds could be larger: at $2100 \mathrm{~K}$, a cloud coverage of up to $50 \%$ would be possible before it is detected by the measurements of this sensitivity. The limits on cool spots are not changed at this temperature (see Fig. 3). If $2 \mathrm{M} 1145$ is cooler $(1700 \mathrm{~K})$, then clouds are limited to being much smaller: a coverage of just $2 \%$ would have given rise to a detectable signature (Fig. 4). Large cool spots, on the other hand, covering up to $40 \%$ of the surface, are permitted. At this lower temperature the dusty models may not be valid (due to increased dust precipitation), so cond models should be considered. For a cond atmosphere at $1700 \mathrm{~K}$, cool spots can still cover a large fraction of the surface (40-60\%) before giving a detectable signature in these bands, but note that these bands were not designed with such a situation in mind. Dusty clouds are restricted to a coverage of no more than $20 \%$. In all cases, these limits apply to a single cloud/spot or several clouds/spots behaving coherently. Clearly, many small features evolving independently of each other will have no net effect on the light curve of the unresolved disk.

These limits can be compared with the feature sizes derivable from the detections of variability in 2M1145 by BJM, specifically the derived amplitudes and noises given in their Table 2 . Using the noise subtraction method in Sect. 6 of the present paper, the 99-01 and 00-02 data from BJM correspond to peak-to-peak signal amplitudes of 0.025 and 0.018 mags respectively. For a dusty atmosphere at $1900 \mathrm{~K}$, these translate to clear cloud coverages of $2.5 \%$ and $2 \%$ respectively, and about the same for $200 \mathrm{~K}$ cooler spots. These are well below the upper limits imposed by the present infrared data and therefore entirely consistent with them.

All of these limits are based on the assumptions (1) that the limiting cases of the AMES-dusty and AMEScond atmospheres of Allard et al. (2001) are appropriate, (2) that 2M1145 was not observed during an abnormal phase of atmospheric or magnetic inactivity, and (3) that the timescale for the evolution of surface features in UCDs is much less than the 54 hour observing period. The case for the latter was argued by BJM.

Clearly, the present work can be improved upon in a number of ways, not least through having a more stable instrument to avoid slit losses. A wider slit may nonetheless be required to achieve pure relative spectrophotometry in order to accommodate brightness changes due to seeing fluctuations. This may require observing in the optical where the sky background is reduced and there are still significant features (see Fig. 2 and Sect. 3.5). While L dwarfs are much fainter here (Fig. 1), this may still be a more effective method if the data quality is limited by systematic errors and not photon noise. Alternatively, $J$ and $K$ band imaging has a significant signature, and has the advantage that many reference stars can be used to calibrate sky variations (see Bailer-Jones \& Lamm 2002). Ultimately, Doppler imaging of the brightest, most rapidly rotating UCDs provides the possibility to monitor surface features in a more direct manner.

\section{Conclusions}

Non-periodic photometric variability in late $M$ and L dwarfs has been indicated by a few observing programs to date. In this paper, I have presented the results of a program to spectrophometrically monitor the known variable L1.5 field dwarf 2M1145 in the near infrared (1$2.5 \mu \mathrm{m})$, in an attempt to identify the cause of its variability. Primary candidates are the temporal evolution of cool magnetically-induced spots and dust-related photospheric clouds. Using the spectral models of Allard et al. (2001), I have demonstrated the photometric signatures of these two types of variability in various bands across the near infrared spectrum. For the evolution of either a clear, dust-precipitated cloud against a dusty atmosphere or a dusty cloud against a dust-cleared atmosphere, there is an anticorrelated variability in the $J$ and $K$ bands. At an effective temperature of $1900 \mathrm{~K}$, the peak-to-peak amplitudes are 0.07 and 0.03 magnitudes respectively for a cloud covering $10 \%$ of the surface, both decreasing by a factor of about three at $2100 \mathrm{~K}$, and the $\mathrm{J}$ band signature increasing by a factor of over five at $1700 \mathrm{~K}$. A cool spot, on the other hand, shows a correlated $J, K$ signature at all temperatures.

The observational data, which cover several hours on three consecutive nights, show no evidence for variability in any of four individual colour indices. However, there 
is evidence for correlated variations in the colours which cannot be attributed to noise or artifacts of the observing method. These changes are more consistent with the presence of a dust-cleared cloud on a dusty atmosphere than a cool dusty spot on such an atmosphere. The nondetections in the individual bands permit upper limits to be placed on the size of any clouds or spots. Adopting a dusty atmosphere for $2 \mathrm{M} 1145$ with an effective temperature of $1900 \mathrm{~K}$, the data restrict clear clouds to cover less than $10-15 \%$ of the surface, or a $200 \mathrm{~K}$ cooler spot (or spots) to cover less than $20 \%$ of the surface. If $2 \mathrm{M} 1145$ is as cool as $1700 \mathrm{~K}$ and still has a dusty atmosphere, then clear clouds are restricted to cover less than $2 \%$ of the surface. However, if a dust-cleared model is more appropriate at $1700 \mathrm{~K}$, the upper limit on cloud coverage is more like $20 \%$.

Acknowledgements. I am grateful to Frank Valdes for advice on some of the more subtle aspects of spectral extraction with IRAF, and to Christiane Helling and Peter Woitke for stimulating conversations on the dynamics of dust in (sub) stellar atmospheres. I would also like to thank France Allard, Peter Hauschildt and Reinhard Mundt for useful comments on a draft of this paper.

\section{References}

Ackerman, A. S., \& Marley, M. S. 2001, ApJ, 556, 872

Allard, F., Hauschildt, P. H., Alexander, D. R., Tamanai, A., \& Schweitzer, A. 2001, ApJ, 556, 357

Bailer-Jones, C. A. L., \& Mundt, R. 1999, A\&A, 348, 800

Bailer-Jones, C. A. L., \& Mundt, R. 2001, A\&A, 367, 218 (BJM), Erratum: A\&A, 374, 1071

Bailer-Jones, C. A. L., \& Lamm, M. 2002, in preparation

Basri, G. 2000, ARA\&A, 38, 485
Basri, G., Mohanty, S., Allard, F., et al. 2000, ApJ, 538, 363

Berger, E. 2002, ApJ, submitted

Berger, E., Ball, S., Becker, K. M., et al. 2001, Nature, 410, 338

Burrows, A., \& Sharp, C. M. 1999, ApJ, 512, 843

Chabrier, G., \& Baraffe, I. 2000, ARA\&A, 38, 337

Chabrier, G., Baraffe, I., Allard, F., \& Hauschildt, P. 2000, ApJ, 542, 464

Clarke, F. J., Tinney, C. G., \& Covey, K. R. 2002, MNRAS, submitted

Fleming, T. A., Giampapa, M. S., \& Schmitt, J. H. M. M. 2000, ApJ, 533, 372

Gizis, J. E., Monet, D. G., Reid, I. N., et al. 2000, AJ, 120, 1085

Gelino, C. R., Marley, M. S., Holtzman, J. A., Ackerman, A. S., \& Lodders, K. 2002, ApJ, submitted

Helling, C., Oevermann, M., Lüttke, M. J. H., Klein, R., \& Sedlmayr, E. 2001, A\&A, 376, 194

Herbst, W., Bailer-Jones, C. A. L., \& Mundt, R. 2001, ApJ, 554, L197

Kirkpatrick, J. D., Reid, I. N., Liebert, J., et al. 1999, ApJ, 519,802

Lodders, K. 1999, ApJ, 519, 793

Marley, M. S., Seager, S., Saumon, D., et al. 2002, ApJ, submitted

Martín, E. L., Zapatero Osorio, M. R., \& Lehto, H. J. 2001, ApJ, 557, 822

Meyer, F., \& Meyer-Hofmeister, E. 1999, A\&A, 341, L23

Nakajima, T., Tsuji, T., Maihara, T., et al. 2000, PASJ, 52, 87

Oliva, E., \& Origlia, L. 1992, A\&A, 254, 466

Reid, I. N., Kirkpatrick, J. D., Gizis, J. E., \& Liebert, J. 1999, ApJ, 527, L105

Schweitzer, A., Gizis, J. E., Hauschildt, P. H., Allard, F., \& Reid, I. N. 2001, ApJ, 555, 368

Tinney, C. G., \& Tolley, A. J. 1999, MNRAS, 304, 119

Tsuji, T. 2001, in Ultracool dwarfs: New Spectral Types L and T, ed. H. R. A. Jones, \& I. Steele (Springer-Verlag, Heidelberg), 9 